\title{
Impact of Interferon-Based Treatment on Quality of Life and Work-Related Productivity of Korean Patients with Chronic Hepatitis C
}

\author{
Sang Hoon $\mathrm{Ahn}^{1}$, Won Hyeok Choe ${ }^{2}$, Yoon Jun $\mathrm{Kim}^{3}$, Jeong Heo ${ }^{4}$, Dorota Latarska-Smuga ${ }^{5}$, Jiho Kang ${ }^{5}$, and Seung Woon \\ Paik $^{6}$ \\ ${ }^{1}$ Department of Internal Medicine, Brain Korea 21 Project for Medical Science, Yonsei University College of Medicine, ${ }^{2}$ Department of \\ Internal Medicine, Konkuk University Hospital, ${ }^{3}$ Department of Internal Medicine and Liver Research Institute, Seoul National University \\ College of Medicine, Seoul, ${ }^{4}$ Department of Internal Medicine, Medical Research Institute, Pusan National University Hospital, Pusan National \\ University College of Medicine, Busan, ${ }^{5} \mathrm{AbbVie}$ Inc., and ${ }^{6}$ Division of Gastroenterology, Department of Medicine, Samsung Medical Center, \\ Sungkyunkwan University School of Medicine, Seoul, Korea
}

Background/Aims: Chronic hepatitis C virus (HCV) infections put patients at risk of serious liver disease and adversely affects patient quality of life (QoL). MOSAIC (International Multicenter Prospective Observational Study to Evaluate the Epidemiology, Humanistic and Economic Outcomes of Treatment for Chronic Hepatitis C Virus) was a prospective, noninterventional, international, multicenter study that aimed to describe the epidemiology of the infection, the impact of the infection on health-related QoL (HRQoL) and daily activities, and healthcare resource use related to HCV and treatment. Here, we present the results on HRQoL and daily activity impairment in consecutively enrolled South Korean patients treated with interferon (IFN)-containing regimens prospectively followed for up to 48 weeks. Methods: General HRQoL, HCV-specific HRQoL, perceived health state, and work/ general activity impairments were measured using the EuroQoL 5-dimension 5-level (EQ-5D-5L), HCV patient-reported outcomes (HCV-PRO), EQ-5D Visual Analog Scale, and Work Productivity and Activity Impairment questionnaires, respectively. Results: Thirty-three of the 100 enrolled patients initiated IFN-based treatment, with an intended duration of 24 weeks for 20 patients and 48 weeks for 12 patients; this information was missing for one patient. Fourteen patients (42.4\%) prematurely withdrew. After treatment initiation, IFNtreated patients showed a trend towards deterioration of both general (baseline: 0.87 \pm 0.103 , week 4: 0.77 \pm 0.153 ) and HCV-specific (baseline: $76.2 \pm 19.5$, week 4: 68.2 \pm 22.3 ) HRQoL. The scores recovered somewhat towards the end of treatment (EOT) $(0.84 \pm 0.146$ for EQ-5D-5L and 70.8 \pm 21.9 for HCV-PRO). The perceived health state and work/general activity impairment displayed similar temporal patterns. Conclusions: Initiating IFN-based treatment prompted some deterioration in general and HCV-related HRQoL, accompanied by impaired daily activities and most work productivity measures; however, the HRQoL and productivity scores improved towards the EOT. HRQoL impairment upon treatment initiation likely contributed to treatment discontinuation. (Gut Liver 2020;14:368-376)

Key Words: Hepatitis C; Interferons; Quality of life

\section{INTRODUCTION}

Chronic infection with the hepatitis C virus (HCV) may lead to the development of serious liver diseases, such as cirrhosis and hepatocellular carcinoma. ${ }^{1}$ Based on the natural history of the disease, it has been estimated that, over 20 to 30 years, up to $20 \%$ of patients develop cirrhosis while $1 \%$ to 5\% may develop hepatocellular carcinoma. ${ }^{1}$ Chronic HCV infection is also known to have a severe negative impact on the patient's quality of life (QoL). ${ }^{2-4}$

The World Health Organization reports that 71 million people worldwide suffer from chronic hepatitis C and almost 400,000 die from the disease every year. ${ }^{5}$ However, actual hepatitis $C$ prevalence may be higher: according to a recent literature review, global prevalence of HCV was estimated at 2.5\%, equating to 177.5 million infected individuals. ${ }^{6}$ In South Korea, the prevalence of $\mathrm{HCV}$ is relatively low-it was estimated at $0.6 \%$

Correspondence to: Seung Woon Paik

Division of Gastroenterology, Department of Medicine, Samsung Medical Center, Sungkyunkwan University School of Medicine, 81 Irwon-ro, Gangnam-gu, Seoul 06351, Korea

Tel: +82-2-3410-3409, Fax: +82-2-3410-6983, E-mail: sw.paik@samsung.com

Received on February 22, 2018. Revised on May 17, 2019. Accepted on May 17, 2019. Published online September 20, 2019. pISSN 1976-2283 eISSN 2005-1212 https://doi.org/10.5009/gnl18100

(c) This is an Open Access article distributed under the terms of the Creative Commons Attribution Non-Commercial License (http://creativecommons.org/licenses/by-nc/4.0) which permits unrestricted non-commercial use, distribution, and reproduction in any medium, provided the original work is properly cited 
to $1.1 \%$ according to a recent review. ${ }^{7}$ Between 2007 and 2011, approximately 60,000 patients per year were diagnosed with hepatitis $\mathrm{C}$ across the country. ${ }^{8}$

HCV infection, particularly a chronic and progressive one, poses a substantial humanistic, clinical and economic burden both to those affected, and to the society as a whole. A retrospective observational study conducted in eight South Korean institutions revealed that, without antiviral treatment, disease progression rate was significantly higher than with treatment among both patients with chronic hepatitis $\mathrm{C}$ and those with compensated cirrhosis. ${ }^{9}$ However, in those whose disease had progressed to the decompensated cirrhosis stage, antiviral treatment did not affect further disease progression. ${ }^{9}$ Hospitalization and outpatient visit requirements increased with disease progression, as did medical costs: the median total medical cost per patient over the 2-year study period ranged from US $\$ 1,179$ for patients with chronic hepatitis C to US\$8,255 in those with hepatocellular carcinoma.

Antiviral treatment is crucial for preventing disease progression. The Korean Association for the Study of the Liver has published Practice Guidelines for Management of Hepatitis C, ${ }^{10}$ providing comprehensive guidance for Korean physicians treating patients infected with HCV. The combination of pegylated interferon $\alpha$ (peg-IFN) and ribavirin (RBV) was the mainstay of chronic hepatitis $\mathrm{C}$ therapy in South Korea before direct-acting antivirals (DAAs) were approved. ${ }^{11}$ DAAs have produced a paradigm shift in the treatment of chronic hepatitis C in Korea, allowing up to $90 \%$ of treated patients to achieve a sustained virological response with minimal adverse events. ${ }^{10}$ Overall the Korean Association for the Study of the Liver guidelines recommend that treatment should be individualized, taking into account a number of factors, such as liver disease severity, probability of treatment success, risks of severe adverse effects, concomitant diseases, and patient preference. ${ }^{10}$

The MOSAIC (International Multicenter Prospective Observational Study to Evaluate the Epidemiology, Humanistic and Economic Outcomes of Treatment for Chronic Hepatitis C Virus) study was conducted to investigate the epidemiology of HCV and provide real-world data on the clinical, humanistic and economic burden of HCV treatment. In this publication, we present the results from the Korean cohort enrolled in this study, focusing on health-related QoL (HRQoL).

\section{MATERIALS AND METHODS}

\section{Objectives}

MOSAIC was a prospective, non-interventional, international, multicenter study. 20 countries including Canada, Russia, Poland, and etc. participated this MOSAIC trial. In South Korea, five sites conducted this study. The study comprised three phases.

The objective of phase 1 (inclusion) was to describe the epi- demiology of HCV infection in South Korea and characterize patients seeking care for HCV. Phases 2 (baseline) and 3 (followup) aimed to: (1) determine how baseline HRQoL and any subsequent HRQoL changes may affect completion of IFN-based treatment; (2) assess the impact of HCV and its treatment on work productivity and activities of daily living; and (3) estimate healthcare resource utilization associated with HCV treatment.

\section{Study design}

The study involved up to five visits: visit 1 in phase 1 , visit 2 in phase 2 and visits 3-5 in phase 3. Phase 1 included consecutive patients with chronic HCV infection who were not undergoing treatment at study inclusion, regardless of HCV genotype, any prior treatment, comorbidities and adjuvant therapies. Demographic and clinical characteristics relevant to chronic hepatitis $\mathrm{C}$ were documented in this first study phase. Participating sites also took note of all patients eligible for inclusion in the study but who were not willing to participate; information on age range and the main reason for not participating was collected for these patients.

The study ended with phase 1 for all patients except those who initiated an IFN-based treatment regimen within 12 weeks of the phase 1 visit, as these patients were included in phases 2 and 3. In phase 2, patients started IFN-based treatment and filled out study questionnaires. Treating physicians provided information on general and HCV-related medical history and prior treatment based on patients' medical records. Information collected at visit 2 (at treatment initiation) served as baseline data for information collected in phase 3. Patients who did not initiate IFN-based treatment within the 12-week time frame were not included. Patients were prospectively followed for up to 48 weeks from the start of treatment (phase 3) over three follow-up visits: the first two at weeks 4 and 12 from visit 2, and the final visit at week 48 or end of treatment (EOT), whichever occurred first. At each visit, patients' clinical status and HCV-related resource utilization were documented, and the patients filled out the study questionnaires listed in Table $1 .^{12-15}$

All treatment, procedures and diagnostic methods followed routine clinical practice. Treatment decisions were solely at the discretion of the treating physician, and all eligible study participants received HCV treatments available on the market, including peg-IFN with or without RBV, either as a single regimen or co-administered with a DAA (telaprevir or boceprevir).

The study was observational and data were collected from all participants, regardless of the precise treatment regimen they received. Informed consent was obtained from all participants included in the study. The study complied with the Ethical Principles for Medical Research Involving Human Subjects outlined in the Helsinki Declaration in 1975 (revised in 2000) and was approved by Institutional Review Boards of the participating centers. 
Table 1. Study Questionnaires

\begin{tabular}{|c|c|c|}
\hline Questionnaire name & Scope & Scoring \\
\hline EQ-5D-5L ${ }^{12,13}$ & General QoL & $\begin{array}{l}\text { Possible index scores range between }-0.109 \text { and 1, with } \\
\text { higher scores indicating better QoL. }\end{array}$ \\
\hline Visual Analogue Scale & Self-rated health state & $\begin{array}{l}\text { Scores range from } 0 \text { to } 100 \text {, with higher scores indicating } \\
\text { better QoL. }\end{array}$ \\
\hline $\mathrm{HCV}-\mathrm{PRO}^{14}$ & Hepatitis C-specific QoL & $\begin{array}{l}\text { Scores range from } 0 \text { to 100, with higher scores indicating } \\
\text { better QoL. }\end{array}$ \\
\hline WPAI $^{15}$ & $\begin{array}{l}\text { WPAI. The questionnaire produces four } \\
\text { scores, each for a different aspect of } \\
\text { work and activity impairment: }\end{array}$ & $\begin{array}{l}\text { The four scores range from } 0 \text { to } 100 \% \text {, with higher per- } \\
\text { centages indicating more severely impaired productiv- } \\
\text { ity. Scores for absenteeism, presenteeism and total work } \\
\text { productivity impairment were not recorded for patients } \\
\text { who were not employed at the time of the assessment. }\end{array}$ \\
\hline Absenteeism & $\begin{array}{l}\text { Percentage of work time missed due to } \\
\text { hepatitis C }\end{array}$ & \\
\hline Presenteeism & $\begin{array}{l}\text { Percentage of impairment due to hepatitis } \\
\text { C while working }\end{array}$ & \\
\hline Total work productivity impairment & Percentage of overall work impairment & \\
\hline Total activity impairment & $\begin{array}{l}\text { Percentage of general (non-work) activity } \\
\text { impairment due to hepatitis }\end{array}$ & \\
\hline
\end{tabular}

EQ-5D-5L, EuroQoL 5-dimension 5-level; HCV-PRO, hepatitis C virus patient-reported outcomes; WPAI, Work Productivity and Activity Impairment questionnaire; QoL, quality of life.

\section{Statistical analysis}

Descriptive statistics were calculated for patient and disease baseline characteristics, and for all QoL assessments. For the latter, five assessment time points are presented, that is, baseline, week 4, week 12, EOT, and week 48. If QoL questionnaires were missing for week 4 or week 12, and the EOT questionnaire of the same patient fell within the time window for week 4 or week 12, the EOT data were also analyzed under that time point. Data in text are presented as mean \pm standard deviation.

\section{RESULTS}

\section{Patient characteristics}

As of the cutoff date (June 30, 2016), 155 Korean patients were recorded in the study database, of whom 55 chose not to participate in the study. As a result, a total of 100 Korean patients were enrolled in phase 1; 86 were treatment-naïve and 14 were previously treated. Of these, 30 treatment-naïve and three treatment-experienced patients initiated IFN-based treatment and were included in phase 2 (see Fig. 1 for a flow diagram of study participants). The three treatment-experienced patients had previously been treated with peg-IFN+RBV $(n=2)$ or other regimens $(n=1)$, but discontinued therapy $(n=2)$, or relapsed $(n=1)$. Mean age of patients participating in phases 2 and 3 was 54.5 years (range, 35 to 71 years) and the majority were female $(\mathrm{n}=19,57.6 \%)$. The predominant HCV genotype was G2 $(\mathrm{n}=19$, $57.6 \%$ ). With regard to liver fibrosis status, this was missing for eight patients (24.2\%), and less than half of the patients $(n=14$, $42.4 \%$ ) had minimal or no liver fibrosis. Baseline and disease characteristics of patients included in phase 2 are shown in Table 2.

\section{Treatment}

Of the 33 patients included in phases 2 and 3, the majority (26 patients [78.8\%], including all three treatment-experienced ones) were treated with the combination of peg-IFN and RBV. Six patients (18.2\%) were treated with the combination of standard IFN and RBV and one patient (3\%) was treated with pegIFN alone, without the addition of RBV. The intended treatment duration was 24 weeks in 20 patients (60.6\%) and 48 weeks in 12 patients; information was missing for one patient. Nineteen patients (57.6\%) completed treatment as planned and 14 (42.4\%) withdrew prematurely (Fig. 1), of whom, four (28.6\%) were lost to follow up or withdrew consent and 10 (71.4\%) withdrew for other reasons. Among these 14 patients, two received IFN and RBV, 11 peg-IFN and RBV, and one peg-IFN alone.

It is worth highlighting that the EOT corresponds to when treatment was prematurely discontinued in some patients, and is not always equivalent to the completion of the full intended treatment period. However, as only five patients (15.2\%) continued treatment until week 48 , we focused the results presentation 


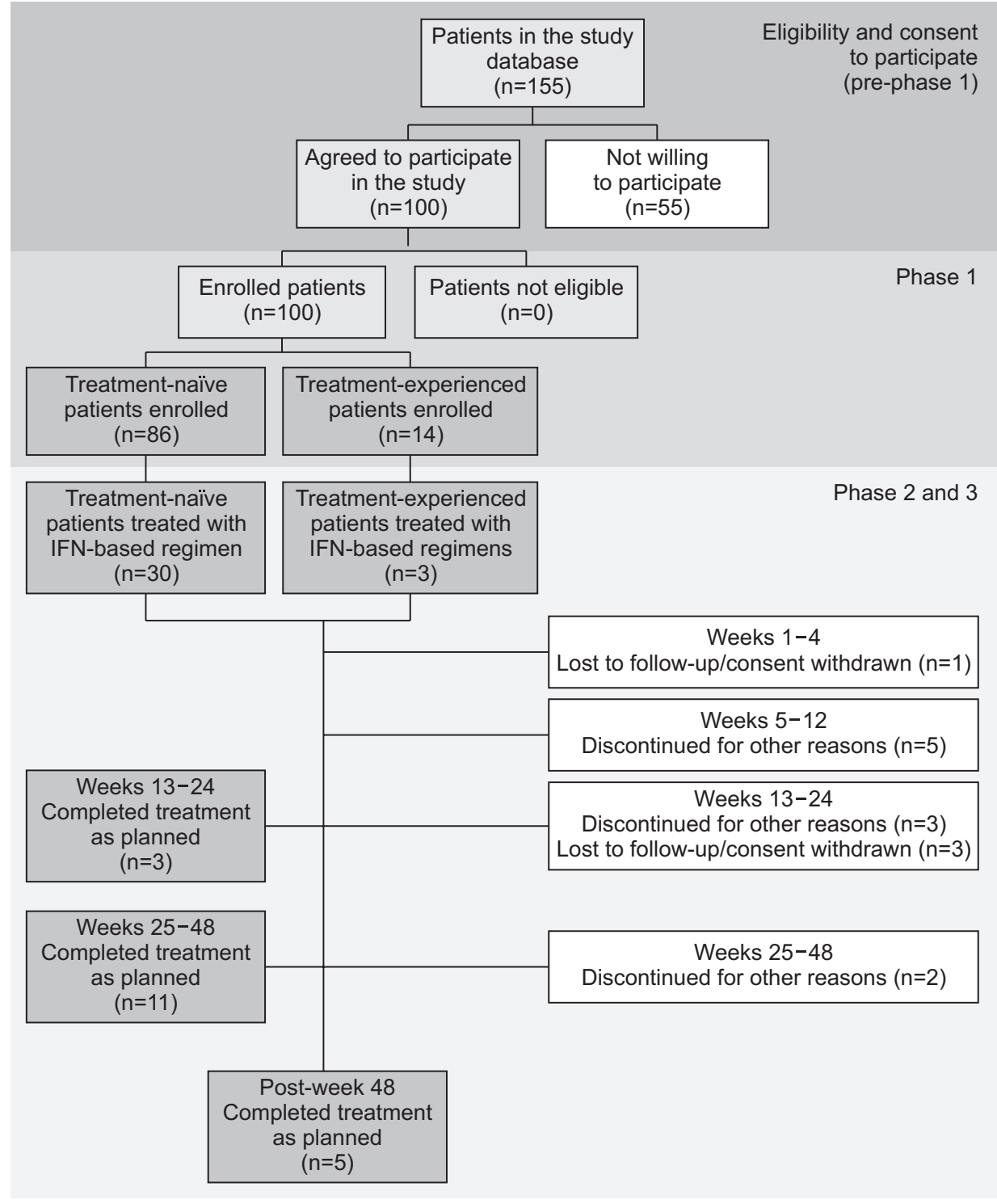

Fig. 1. Flow diagram of study participants. IFN, interferon. on the change in QoL and productivity from baseline to the EOT, with week 48 data shown for information only.

Among 33 patients with enrolled phase 2 of this study, 22 patients were achieved sustained viral response rate at 12 weeks (SVR12) after treatment $(66.7 \%, 22 / 33)$. The SVR12 of patient of genotype 1 was $63.6 \%$ (7/11) and SVR12 of genotype 2 was $68.2 \%(15 / 22)$ respectively.

\section{Health-related QoL}

After treatment initiation, IFN-treated patients showed a slight trend for deterioration of both general and HCV-specific HRQoL, measured using EuroQoL 5-dimension 5-level (EQ-5D5L) and HCV patient-reported outcomes (HCV-PRO), respectively. All 33 patients filled in the EQ-5D-5L at baseline and the mean score was $0.87 \pm 0.103$. Mean scores were substantially lower at weeks 4 and 12, but at the EOT they partially recovered reaching on average $0.836 \pm 0.146$ across the 23 patients who filled in the questionnaire (Fig. 2). In the four patients who continued treatment until week 48 , the EQ-5D-5L score at week 48 neared the baseline value, with a mean of $0.867 \pm 0.089$. The mean decreases in EQ-5D-5L score from baseline to the EOT was $0.03 \pm 0.18$ when all available data for the two time points were used. When only patients with available data for both time points (i.e., baseline and week 4 , baseline and week 12 , etc.) were included in the calculation, a similar temporal pattern was also observed, with scores decreasing noticeably from baseline in weeks 4 and 12 to recover somewhat at the EOT (data not shown).

As part of the EQ-5D-5L questionnaire, patients also marked their perceived health state on a visual analogue scale (VAS). When all available data was used, the mean health state rating at baseline was $78 \pm 13.5(n=33)$, but, similar to the general EQ5D-5L score, it decreased after patients initiated treatment with IFN and then largely recovered by the EOT, reaching $75 \pm 14.9$ 
Table 2. Baseline Patient and Disease Characteristics Stratified by Treatment Group

\begin{tabular}{lc}
\hline \multicolumn{1}{c}{ Patients in phase 2 } & Value (n=33) \\
\hline Sex & $14(42.4)$ \\
Male & $19(57.6)$ \\
Female & $54.5(35-71)$ \\
Age, yr & \\
Detailed liver fibrosis stage & \\
(excluding cases with unknown status) & $14(56.0)$ \\
Minimal or no fibrosis & $2(8.0)$ \\
Portal fibrosis & $3(12.0)$ \\
Bridging (or septal) fibrosis & $6(24.0)$ \\
Cirrhosis & $8(32.0)$ \\
Unknown & \\
Hepatitis C virus genotype & $11(33.3)$ \\
G1 & $19(57.6)$ \\
G2 & $3(9.1)$ \\
G3 & $2(6.1)$ \\
Extrahepatic manifestations present
\end{tabular}

Data are presented number (\%) or mean (range).

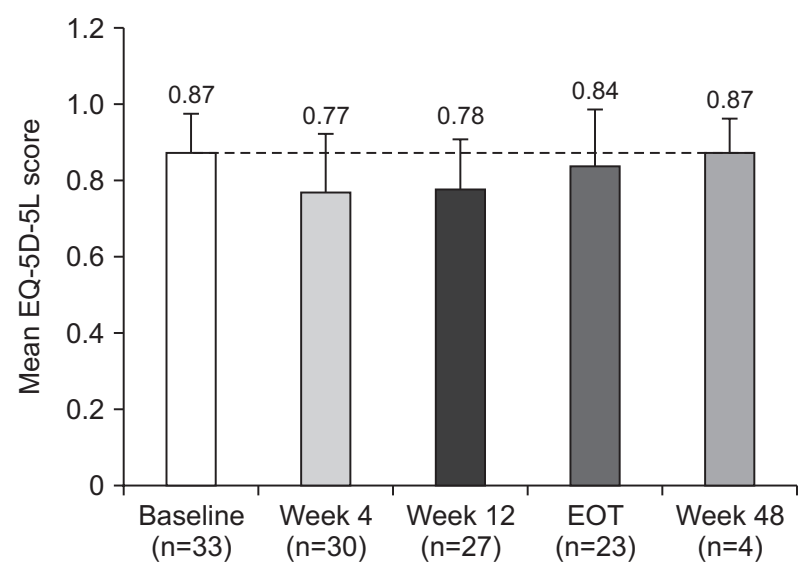

Fig. 2. EQ-5D-5L scores over the treatment period. EQ-5D-5L, EuroQoL 5-dimension 5-level; EOT, end of treatment.

on average $(\mathrm{n}=23)$ (Fig. 3).

Considering only patients with available data at both time points, there was a significant decrease in the EQ-5D VAS score from baseline to week 4 ( $\mathrm{n}=28,77.5$ vs 67.7 , respectively, $\mathrm{p}=0.003$ two-sided paired t-test) and from baseline to week 12 ( $n=26,78.1$ vs 70.6, respectively, $\mathrm{p}=0.006$ two-sided paired ttest). However, there was no statistically significant difference between EQ-5D VAS scores at baseline and at EOT $(n=23,77.2$ vs $75.4, p=0.621$ two-sided paired t-test). These comparing result among the 22 patients who achieved SVR12 was same as well, except between week 12 and EOT. The EQ-5D VAS scores showed statistically significant increase at EOT compare to week 12 ( $\mathrm{n}=18,73.3$ vs 78.3, respectively, $\mathrm{p}=0.011$ two-sided paired t-

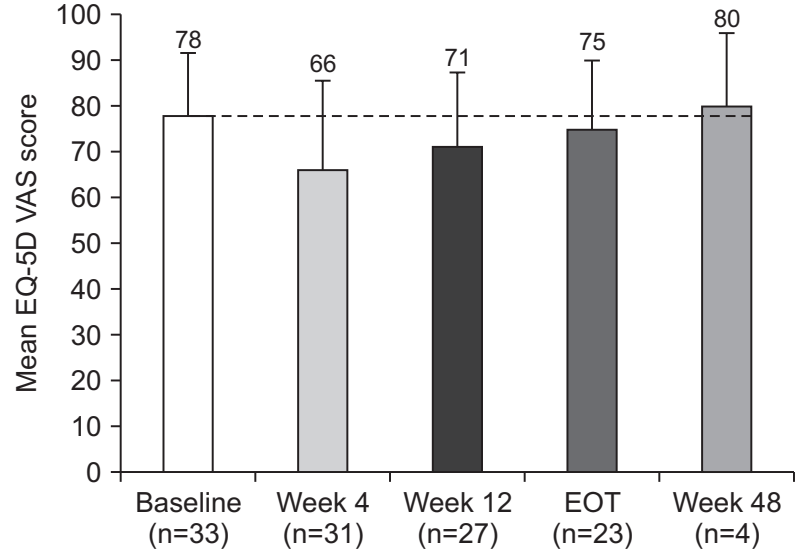

Fig. 3. Self-rated health state recorded on the EQ-5D VAS. EQ-5D, EuroQoL 5-dimension; VAS, visual analogue scale; EOT, end of treatment.

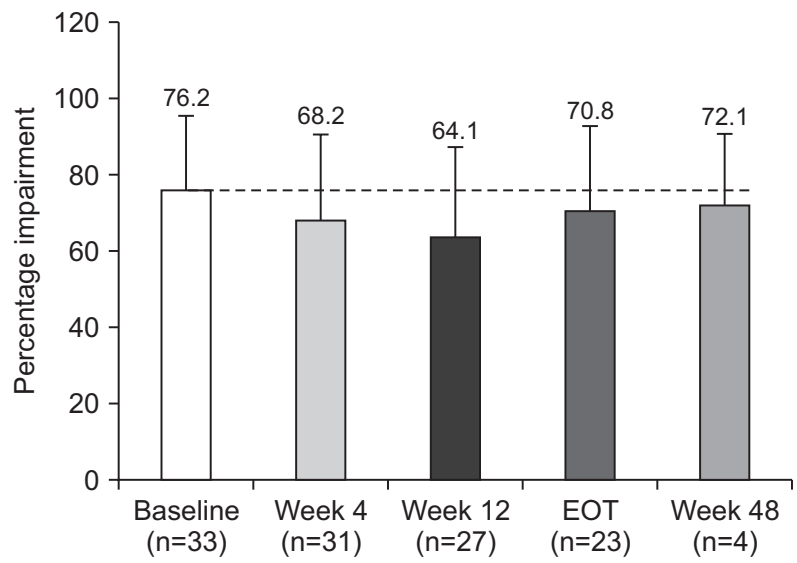

Fig. 4. HCV-PRO scores over the treatment period.

HCV-PRO, hepatitis C virus patient-reported outcomes; EOT, end of treatment.

test).

In terms of HCV-specific QoL aspects measured using HCVPRO (Fig. 4), the mean baseline score among all 33 patients participating in the study was $76.2 \pm 19.5$. Similar to the general QoL (EQ-5D-5L) scores, the average HCV-PRO score decreased at week 4. This decrease continued through week 12 but scores recovered partially at the EOT, reaching on average $70.8 \pm 21.9$ in the 23 patients who responded. A similar near-recovery of the HCV-PRO score was observed at week 48 in patients who continued treatment until that point $(72.1 \pm 18.8, n=4)$. The mean decrease in HCV-PRO score from baseline to the EOT was $5.40 \pm 29.32$ when all available data for the two time points were used. Considering only patients with available data at both time points, the pattern of responses over time was similar (data not shown).

Korean patients treated with IFN also noted impairment in work productivity and general activities, as measured using the Work Productivity and Activity Impairment (WPAI) questionnaire (Table 3, Fig. 5). The results for the former measure were, 
Table 3. WPAI Scores at the Consecutive Study Visits

\begin{tabular}{lccccc}
\hline \multicolumn{1}{c}{ Measure } & Baseline & 4 Weeks & 12 Weeks & EOT & 48 Weeks $^{*}$ \\
\hline Absenteeism, \% & $2.2 \pm 7.9(\mathrm{n}=13)$ & $12.9 \pm 22.2(\mathrm{n}=11)$ & $6.1 \pm 13.0(\mathrm{n}=10)$ & $2.2 \pm 4.4(\mathrm{n}=9)$ & $0.0(\mathrm{n}=1)$ \\
Presenteeism, \% & $22.1 \pm 29.4(\mathrm{n}=14)$ & $38.3 \pm 27.2(\mathrm{n}=12)$ & $40.0 \pm 28.3(\mathrm{n}=10)$ & $26.0 \pm 28.4(\mathrm{n}=10)$ & $60.0(\mathrm{n}=1)$ \\
Total work productivity impairment, \% & $25.1 \pm 30.3(\mathrm{n}=13)$ & $44.1 \pm 32.8(\mathrm{n}=11)$ & $44.1 \pm 28.2(\mathrm{n}=10)$ & $22.5 \pm 26.1(\mathrm{n}=9)$ & $60.0(\mathrm{n}=1)$ \\
Total activity impairment, \% & $24.7 \pm 26.0(\mathrm{n}=32)$ & $37.1 \pm 31.1(\mathrm{n}=31)$ & $46.3 \pm 29.6(\mathrm{n}=27)$ & $37.8 \pm 30.3(\mathrm{n}=23)$ & $40.0 \pm 31.6(\mathrm{n}=4)$ \\
\hline
\end{tabular}

Data are presented as mean \pm SD.

WPAI, Work Productivity and Activity Impairment questionnaire; EOT, end of treatment.

*Only a single patient who continued treatment until week 48 was included. Consequently, the values for absenteeism, presenteeism and total work productivity impairment at week 48 are those reported by that patient.

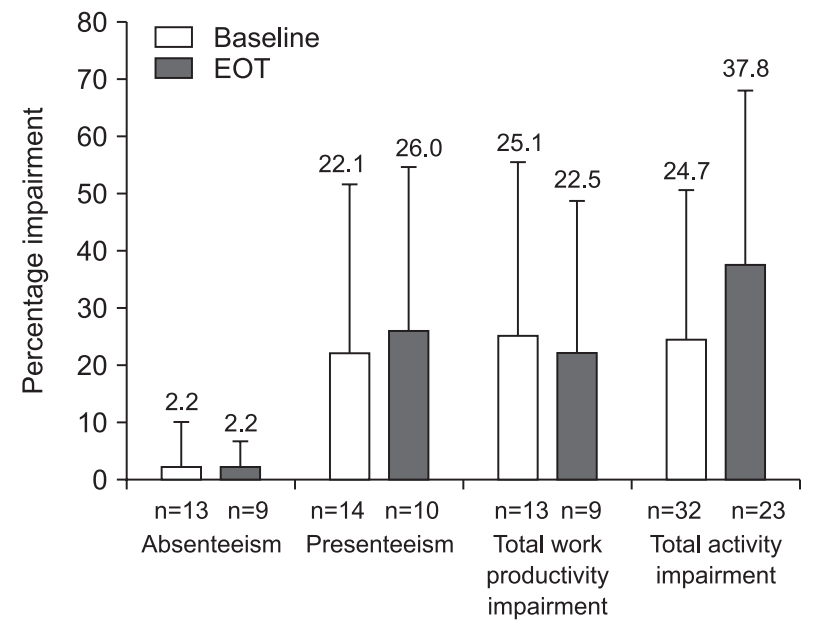

Fig. 5. WPAI scores at baseline and at end of treatment (EOT). WPAI, Work Productivity and Activity Impairment questionnaire.

however, limited to patients who were employed at the time of the assessment (15 patients, at baseline; 12, at week 4; 10, at week 12; one, at week 48 and 10 at EOT). From a baseline average of $22.1 \% \pm 29.4 \%$ across 14 patients, the estimated percentage of impairment due to hepatitis $\mathrm{C}$ while working (presenteeism) increased slightly (by 3.9\% $\pm 40.9 \%$ ) to reach $26.0 \% \pm 28.4 \%$ among 10 respondents at the EOT. On the other hand, absenteeism (i.e., the percentage of work time missed due to hepatitis $\mathrm{C}$ ) hardly changed between baseline $(2.2 \% \pm 7.9 \%, n=13)$ and the EOT $(2.2 \% \pm 4.4 \%, n=9)$, with a difference of $0.0 \% \pm 9.0 \%$ between these two time points. Finally, total work productivity impairment decreased very slightly (by $2.6 \% \pm 40.0 \%$ ) between baseline $(25.1 \% \pm 30.3 \%, n=13)$ and the EOT $(22.5 \% \pm 26.1 \%, n=9)$. Importantly, patients noted increased impairment in all three aspects of work productivity after treatment initiation (week 4) (Table 3), but this largely stabilized or even started to improve at week 12 , with scores recovering-at least to some extent-at the EOT (Table 3, Fig. 5). When only data from patients with available information at both time points were analyzed, the temporal patterns of responses were similar for presenteeism and absenteeism, although patients actually reported a decrease (by 3.0\% $\pm 7.29 \%$, $\mathrm{n}=6$ ) in the degree of absenteeism at the EOT compared with baseline (data not shown). In terms of total work productivity impairment, however, the pattern was slightly different than when all available data were used, in that the increased impairment of baseline productivity observed at weeks 4 and 12 persisted almost unchanged at the EOT, suggesting that some patients may be prone to experiencing sustained adverse effects associated with treatment (data not shown). It is worth noting that the results related to work productivity should be interpreted with caution, as they arise from a limited number of patients.

The final aspect of impairment measured using WPAI was total activity impairment, that is, the percentage of general (non-work) impairment due to hepatitis. Thirty-two out of 33 patients participating in the study provided baseline data for this measure, with an average total activity impairment of $24.7 \% \pm 26.0 \%$. At the EOT, average total activity impairment was 37.8\% $\pm 30.3 \%$ among 23 patients, which corresponded to a $13.1 \% \pm 39.9 \%$ increase in impairment from baseline, suggesting a deterioration of the ability to perform daily activities (Fig. 5). A similarly high degree of impairment was observed at week 48 in the four patients who continued treatment until that point (40.0\% $\pm 31.6 \%)$. Impairment in general activity followed a similar temporal pattern to that seen with work-related impairment, with baseline impairment worsening once IFN-based treatment was initiated (week 4), a further increase in impairment at week 12, and then a decline towards the EOT (Table 3). A similar pattern was observed when only patients with available data for both time points contributed to the analysis (data not shown).

\section{DISCUSSION}

Probably the most crucial result observed in Korean patients with a HCV infection initiating IFN-based treatment was a clear trend for decreased QoL and productivity following treatment initiation. Although the QoL and productivity estimates slowly improved over a longer treatment period, they often did so incompletely.

A systematic review and pooled analysis of data on QoL of HCV patients identified 15 studies comparing HRQoL between HCV patients and healthy controls. ${ }^{4}$ These studies used the SF36 Health Survey rather than the EQ-5D, so the data cannot be compared directly with our results; however, HCV was shown 
to have a moderate to large adverse impact on all QoL aspects measured, especially social and physical function, general health, and vitality. ${ }^{4}$ Some of that QoL impairment is likely to be due to the disease itself, and can be seen before treatment initiation. Indeed, analysis of data from 324 patients receiving IFN for hepatitis $\mathrm{C}$ relapse in an international study revealed that, compared with the U.S. general population, chronic hepatitis C patients experienced impairments in five out of eight QoL aspects even before treatment was initiated, ${ }^{3}$ suggesting that the disease itself poses a burden on the patients, adversely affecting their QoL. Similar results were seen among 642 North American patients in a multicenter, double-blind, controlled clinical trial of IFN, where pretreatment QoL of chronic hepatitis C patients was worse than that of the U.S. general population in terms of all eight QoL aspects studied. ${ }^{2}$ Similarly, in our study, pretreatment (baseline) EQ-5D-5L score $(0.87 \pm 0.103)$ was somewhat lower compared with Korean population norm (mean \pm standard error: 0.958 \pm 0.002$).{ }^{16}$ It is, however, worth noting that the general population score quoted above was obtained using the EQ5D-3L questionnaire, ${ }^{16}$ and no population norm is available for EQ-5D-5L in Korea.

The baseline EQ-5D-VAS score $(78 \pm 13.5)$ was almost the same as the Korean population norm (mean \pm standard error: $79.5 \pm 0.4) .{ }^{16}$ However, the normative data are based on a 2009 study $^{16}$ and a more recent publication with a similar sample size reported an average EQ-5D-VAS score of $83 \pm 10$ for a Korean general population administered the EQ-5D-5L. ${ }^{17}$ Compared with the latter general population score, ${ }^{17} \mathrm{HCV}$ patients in our study noted a 5-point impairment in perceived health state before starting their treatment.

In addition to the impairment in general QoL, patients also reported impairments in work and general productivity at baseline. Although they tended not to be absent from work due to hepatitis, perceived impairment while working (presenteeism), overall work impairment and impairment of non-work activities reached, on average, approximately 25\% before the start of treatment.

IFN-based treatment of chronic hepatitis $\mathrm{C}$ has further adverse impact on patients' QoL. Evidence from the aforementioned international study of relapsed hepatitis $\mathrm{C}$ patients pointed to a decline in patient QoL during IFN-based treatment, especially at the start of treatment, with some improvement seen already at 24 weeks of treatment and the scores returning to baseline over 24 weeks posttreatment. ${ }^{3}$ A similar pattern was observed in our study, although we did not investigate posttreatment values. This improvement in QoL with continued treatment suggests patients may have developed a coping mechanism, and become somewhat used to the adverse effects of IFN and RBV. Alternatively, it is possible that patients who particularly poorly tolerated treatment discontinued it early, contributing to the high discontinuation rate observed. Thus, these patients-whose scores could be expected to be lower compared with patients who tolerated treatment well-would not contribute to the average QoL and productivity scores at later time points, resulting in improvements in scores over time. Importantly, the decline in HRQoL during treatment in our study was evident despite nearly $80 \%$ of patients receiving peg-IFN, which has been shown to produce less QoL impairment in hepatitis C patients compared with standard IFN. ${ }^{18,19}$ However, the fact that all patients but one received RBV likely contributed to the QoL impairment, as the addition of RBV to IFN has been shown to reduce QoL. ${ }^{18}$

The improvement in QoL scores towards the EOT may also be associated with virological response, as viral RNA was undetectable in 23 patients (68.7\%) at 48 weeks or EOT and in 22 patients $(66.7 \%)$ at $\geq 12$ weeks posttreatment. In a number of studies, good virological response to treatment has been shown to associate with improvements in QoL compared with nonresponders. ${ }^{2-4,18,19}$ Although the attainment of good virological response could have contributed to the improvements in QoL seen over time in our study, we are unable to determine this due to the large amount of missing virological response data during the treatment period, ranging from five patients (15.2\%) at week 48 or EOT to as many as $28(84.8 \%)$ at week 24 and 32 (97.0\%) at week 36. Nonetheless, the fact that EQ-5D VAS results were similar in patients who achieved SVR12 and the overall study population suggests that on-treatment HRQoL is far more strongly affected by adverse effect of the treatment than its outcome.

A high number of patients ( $\mathrm{n}=14,42.4 \%)$ in our study withdrew prematurely. A pooled analysis of data from 1,441 patients enrolled in three international, multicenter randomized clinical trials of IFN reported a lower withdrawal rate, with only 10\% of patients discontinuing treatment before week 24, but showed that worsening fatigue and deteriorating QoL significantly predicted premature discontinuation. ${ }^{19}$ With the World Health Organization reporting only half of chronic disease patients in the developed countries adhere to their medication, ${ }^{20}$ adherence can be seen as an issue common to many chronic diseases. In chronic hepatitis $\mathrm{C}$, however, novel DAAs with minimal adverse events may improve patient adherence to therapy, providing hopes for better response to treatment.

MOSAIC was a non-interventional, real-world study, capturing everyday clinical practice related to HCV treatment in South Korea. As such, it examined routine care, making the results more readily applicable to standard treatment of HCV patients seen in the clinic outside the trial setting. Undoubtedly, comprehensive and thorough QoL assessment is one of the greatest strengths of the MOSAIC study. We used validated instruments to assess health state and general HRQoL (EQ-5D), and HCVspecific QoL aspects (HCV-PRO). Further, we investigated the impact of treatment on daily life and productivity using WPAIalso a well-validated instrument. However, the study is not without limitations: the small population of treated patients (phases 2 and 3) combined with a high withdrawal rate means 
many patients did not complete their treatment as planned. The results from week 48 arise from a particularly small number of patients. Although 48 weeks was the intended treatment duration for just over a third of the study patients, the small population size compounded by a high withdrawal rate meant only five patients completed 48 weeks of treatment. The small population size also particularly impacted work-related productivity measures, as these were only applicable to patients who were employed, that is, less than $50 \%$ of the study population at baseline. Another important limitation relates to the fact that information on doses of IFN, peg-IFN and RBV administered to each patient was not collected in this study. It is therefore possible that the HRQoL improvement observed towards the EOT could be attributed to adjustment of treatment doses. However, the focus of this study was on the longitudinal QoL change during and after treatment, rather than on dose-QoL relationship. Hence, the results of MOSAIC, like those of any observational study, should be interpreted to imply association and not causality.

Following treatment initiation, the Korean cohort of IFNtreated patients with chronic hepatitis $\mathrm{C}$ enrolled in the MOSAIC study experienced some deterioration of both general and HCVrelated HRQoL, accompanied by impairment of daily activities and most work productivity measures. However, towards the end of treatment most QoL and productivity scores improved markedly, often nearing pretreatment values. The QoL impairment seen upon treatment initiation likely contributed to the high treatment discontinuation rate observed. New DAAs developed to target chronic hepatitis $\mathrm{C}$ should address this issue, aiming for HRQoL and productivity improvement in treated patients, which could improve adherence, ultimately improving patients' chances of recovery.

\section{CONFLICTS OF INTEREST}

S.H.A. has served as an advisor and lecturer for Bristol-Myers Squibb, Gilead Sciences, F. Hoffmann-La Roche, Merck, AbbVie, and has received unrestricted grants from Bristol-Myers Squibb, Gilead Sciences, and F. Hoffmann-La Roche for investigatorinitiated trials. J.H. has received a grant from GSK, and research support from BMS and Roche; also acted as an advisor to Abbvie, BMS, Gilead Sciences, Pharma Essentia, SillaJen, and Johnson \& Johnson. D.L.S. and J.K. are employees of AbbVie, Inc. and may hold stock or stock options. S.W.P. has received grant and research support from AbbVie, BMS, Gilead, GSK, Merck, Novartis, and Roche. W.H.C. and Y.J.K have no conflicts of interest to declare.

\section{ACKNOWLEDGMENTS}

The design, conduct, analysis, and financial sponsorship of the MOSAIC study were provided by AbbVie. AbbVie partici- pated in the interpretation of data, review and approval of the content of this manuscript. Medical writing support was provided by Proper Medical Writing Sp. z o.o. and funded by AbbVie.

\section{AUTHOR CONTRIBUTIONS}

Study concept and design: all authors. Data acquisition: S.H.A., W.H.C., Y.J.K., J.H., S.W.P. Data analysis and interpretation: all authors. Drafting of the manuscript: D.L.S., J.K. Critical revision of the manuscript for important intellectual content: all authors. Statistical analysis: D.L.S., J.K. Administrative, technical, or material support; study supervision: D.L.S., J.K.

\section{ORCID}

Sang Hoon Ahn https://orcid.org/0000-0002-3629-4624 Won Hyeok Choe https://orcid.org/0000-0002-8019-5412 Yoon Jun Kim https://orcid.org/0000-0001-9141-7773 Jeong Heo Jiho Kang https://orcid.org/0000-0003-0961-7851 Seung Woon Paik https://orcid.org/0000-0002-8208-2243 https://orcid.org/0000-0002-6746-6652

\section{REFERENCES}

1. Sebastiani G, Gkouvatsos K, Pantopoulos K. Chronic hepatitis C and liver fibrosis. World J Gastroenterol 2014;20:11033-11053.

2. Bonkovsky HL, Woolley JM. Reduction of health-related quality of life in chronic hepatitis $\mathrm{C}$ and improvement with interferon therapy. The Consensus Interferon Study Group. Hepatology 1999;29:264-270.

3. Ware JE Jr, Bayliss MS, Mannocchia M, Davis GL. Health-related quality of life in chronic hepatitis C: impact of disease and treatment response. The Interventional Therapy Group. Hepatology 1999;30:550-555.

4. Spiegel BM, Younossi ZM, Hays RD, Revicki D, Robbins S, Kanwal F. Impact of hepatitis $C$ on health related quality of life: a systematic review and quantitative assessment. Hepatology 2005;41:790800.

5. World Health Organization (WHO). Hepatitis C: fact sheet [Internet]. Geneva: WHO; c2010 [cited 2017 Nov 5]. Available from: http:// www.who.int/mediacentre/factsheets/fs164/en/.

6. Petruzziello A, Marigliano S, Loquercio G, Cozzolino A, Cacciapuoti C. Global epidemiology of hepatitis $\mathrm{C}$ virus infection: an up-date of the distribution and circulation of hepatitis $C$ virus genotypes. World J Gastroenterol 2016;22:7824-7840.

7. Bennett H, Waser N, Johnston K, et al. A review of the burden of hepatitis $\mathrm{C}$ virus infection in China, Japan, South Korea and Taiwan. Hepatol Int 2015;9:378-390.

8. Kwon GY, Lee H, Gwack J, Lee SW, Ki M, Youn SK. Regional distribution of hepatitis $\mathrm{C}$ virus infection in the Republic of Korea 2007-2011. Gut Liver 2014;8:428-432.

9. Kim do Y, Yoon KT, Kim W, et al. Estimation of direct medical 
cost related to the management of chronic hepatitis $\mathrm{C}$ and its complications in South Korea. Medicine (Baltimore) 2016;95:e3896.

10. Korean Association for the Study of the Liver. KASL clinical practice guidelines: management of hepatitis C. Clin Mol Hepatol 2016;22:76-139.

11. Korean Association for the Study of the Liver (KASL). KASL clinical practice guidelines: management of hepatitis C. Clin Mol Hepatol 2014;20:89-136.

12. Herdman M, Gudex C, Lloyd A, et al. Development and preliminary testing of the new five-level version of EQ-5D (EQ-5D-5L). Qual Life Res 2011;20:1727-1736.

13. Janssen MF, Pickard AS, Golicki D, et al. Measurement properties of the EQ-5D-5L compared to the EQ-5D-3L across eight patient groups: a multi-country study. Qual Life Res 2013;22:1717-1727.

14. Anderson RT, Baran RW, Dietz B, Kallwitz E, Erickson P, Revicki DA. Development and initial psychometric evaluation of the hepatitis C virus-patient-reported outcomes (HCV-PRO) instrument. Qual Life Res 2014;23:561-570.
15. Reilly MC, Zbrozek AS, Dukes EM. The validity and reproducibility of a Work Productivity and Activity Impairment instrument. Pharmacoeconomics 1993;4:353-365.

16. Szende A, Janssen B, Cabases J. Self-reported population health: an international perspective based on EQ-5D. Dordrecht: Springer, 2014.

17. Kim SH, Ahn J, Ock M, et al. The EQ-5D-5L valuation study in Korea. Qual Life Res 2016;25:1845-1852.

18. Hassanein T, Cooksley G, Sulkowski M, et al. The impact of peginterferon alfa-2a plus ribavirin combination therapy on health-related quality of life in chronic hepatitis C. J Hepatol 2004;40:675681.

19. Bernstein D, Kleinman L, Barker CM, Revicki DA, Green J. Relationship of health-related quality of life to treatment adherence and sustained response in chronic hepatitis $\mathrm{C}$ patients. Hepatology 2002;35:704-708.

20. Sabaté E. Adherence to long-term therapies: evidence for action. Geneva: World Health Organization, 2003. 